\title{
Music Therapy during End-of-Life Care in the Neonatal Intensive Care Unit (NICU) - Reflections From Early Clinical Practice in Colombia
}

\author{
Mark Ettenberger ${ }^{12}$ * \\ 1 SONO - Centro de Musicoterapia \\ 2 Universidad Nacional de Colombia \\ *mark.ettenberger@gmx.at
}

Received: 25 April 2017 ; Accepted: 1 June 2017 ; Published: 1 July 2017

Editor: Daphne Rickson Reviewers: Monika Nöcker-Ribaupierre, Elizabeth Mclean

\begin{abstract}
Music therapy during end-of-life care has become an increasingly applied therapeutic option for a broad range of populations in a variety of settings. While music therapy in the Neonatal Intensive Care Unit (NICU) is a well-established field of clinical research and practice, there is a lack of literature that specifically addresses issues related to end-of-life care in this setting. In this article, the author aims to reflect upon some of the potentials, difficulties, and challenges when accompanying babies and their families during their last stages of life in the NICU. Three case vignettes will be described in order to exemplify aspects related to rituality, culture, memory making, and family-centred care. It is hoped that these case studies prompt the exchange of ideas and experiences among music therapists and other health care professionals concerned with end-of-life care in the NICU.
\end{abstract}

Keywords: end-of-life care, music therapy, NICU, preterm infants, palliative care, family-centred care, Colombia

\section{Introduction}

Another plausible model for an origin of early human music is the lament, a widespread performance by individuals or groups in which the natural behaviour of weeping and moaning in grief at the loss by death or separation from a loved one became formalized and elaborated in song/poetry/movement, and shared with others to relieve feelings of helplessness, individual isolation, despair, and the anxiety attendant on the interruption death makes to the life of an individual or group. (Dissanayke, 2009, p. 26)

The death of a young child or baby is certainly one of the most devastating events for the affected families and can be a highly demanding and sensitive issue for health care professionals. As a music therapist working for some years in the Neonatal Intensive Care Unit (NICU), I had the chance to accompany several babies and their families during their last stages of life. These experiences were incredibly enriching from both a professional and personal point of view but also quite challenging. The aim of this article is to reflect upon some of the encountered potentials and difficulties when 
providing music therapy during end-of-life care in the NICU and to encourage further discussion among health care professionals involved in neonatal care.

Over the last decades, music therapy in the NICU has become an expanding area of clinical research and practice, focusing on the wellbeing of both the babies and their parents (for an overview of research studies, see for example Bieleninik, Ghetti, \& Gold, 2016; Haslbeck, 2012; Hartling et al., 2009; Hodges \& Wilson, 2010; Krueger, 2010; Standley, 2012; van der Heijden et al., 2016). However, while music therapy is widely applied for a variety of populations with terminal diseases including paediatrics (e.g. Archie, Bruera, \& Cohen, 2013; Bradt \& Dileo, 2010; Hilliard, 2005; McConnell, Scott, \& Porter, 2016), no publications were found that specifically address music therapy during end-of-life care in the NICU. This suggests that it may not yet be a fully integrated aspect of clinical practice or a currently active field of research. Nonetheless, parental grief is a topic that is cautiously emerging in recent music therapy writings (Haslbeck, 2016) and single case studies about end-of-life care in the NICU are reported in Ettenberger (2017a; 2017b in press). Also, from discussions with several colleagues from around the world it becomes clear that there is a growing interest and concern about music therapy during end-of-life care in the NICU, for which this article presents some first experiences from our early clinical practice.

The most fundamental aims of end-of-life care are to support patients during their final months or years of life and to help them to die in dignity (NHS, 2016). Dying with dignity "recognizes the intrinsic, unconditional quality of human worth but also external qualities of physical comfort, autonomy, meaningfulness, preparedness, and interpersonal connection" (Cook \& Rocker, 2014, p. 2506). End-of-life care is ideally provided by a multidisciplinary team of caregivers, including specially trained physicians, nurses, therapists, counsellors, social workers, chaplains, among others (National Consensus Project for Quality Palliative Care, 2013). Palliative care - as an integral part of end-of-life care - "is an approach that improves the quality of life of patients and their families facing the problem associated with life-threatening illness, through the prevention and relief of suffering by means of early identification and impeccable assessment and treatment of pain and other problems, physical, psychosocial and spiritual" (WHO, 2016). This definition is clearly in line with the goals of music therapy during end-of-life care, such as helping patients in addressing their physical, emotional, and spiritual needs, improving their quality of life, and assisting patients and caregivers in coping and bereavement (Bradt \& Dileo, 2010). While the need for more evidence in this field is suggested, a growing number of research studies and clinical reports indicate that music therapy can help palliative care patients to alleviate emotional distress, improve quality of life and physical comfort, decrease pain levels, fatigue, and drowsiness, and successfully address spiritual needs of both patients and their families (e.g. Forrest, 2014; Gutgsell et al., 2013; Horne-Thompson \& Grocke, 2008; Kordovan et al., 2016; O'Kelly, 2002).

While much of the existing literature on this subject is related to cancer care, supporting patients with a life-threatening illness is also an increasingly discussed topic in general Intensive Care Units (ICUs). This is highly relevant considering that for example in the United States about $22 \%$ of all deaths occur in the ICU (Papadimos et al., 2011). Recent reviews and guidelines about palliative care in ICUs include recommendations regarding communication with the families, decision-making processes, and stress the need to consider cultural aspects as well as the spiritual beliefs and traditions of the affected patients and families (Cook \& Rocker, 2014; Luce, 2010; Papadimos et al., 2011; Truog et al., 2008). With regard to paediatric and neonatal populations, such guidelines also mention the use of music and music therapy as beneficial strategies for improving the infants' quality of life, for fostering family engagement, and for reducing pain and promoting comfort (AAP, 2000, 2013; Mancini et al., 2014). This may suggest acceptability of music therapy during end-of-life care in ICUs also for non-cancer related diseases, such as it is the case of the NICU.

For this article, it was opted to present three distinctive case vignettes. The cases were chosen with the aim to reflect the diversity of possible scenarios for music thera- 
pists working in this field: from a several months long process in the first vignette, to accompanying a baby that initially was not referred to, but transitioned to end-of-life care during hospitalization in the second case, to a single focal session in the third case. Contrary to other populations, research about music therapy in the NICU has predominantly focused on quantitative designs so far and it was not until very recently that the first qualitative studies (Haslbeck, 2013; Ullsten et al., 2017) or mixed-methods studies (Ettenberger et al., 2014; Ettenberger et al., 2016) were published. More qualitative approaches including case-study reports are therefore vital, since they are the building blocks for theory development and an important element for gaining insight into the reality of (clinical) practice (Flyvbjerg, 2006). This may be especially relevant considering the on-going internationalization of music therapy in the NICU (Shoemark, 2015). Before introducing the case vignettes, a short description of our music therapy service will be provided in order to understand better the organizational aspects in which the clinical work takes place.

\section{The NICU of the Centro Policlínico del Olaya (CPO)}

The NICU of the CPO is situated in Bogotá, the capital of Colombia. It is a Level III facility consisting of 28 beds distributed on two floors for both intensive and intermediate care. The NICU is a semi-open unit, which means that parents cannot stay overnight. Visiting hours are normally from $8 \mathrm{am}-6 \mathrm{pm}$. Music therapy is part of an interdisciplinary therapy team that includes respiratory therapists, an occupational therapist and a speech and language therapist. Psychological support is available for parents throughout the hospital, although no palliative care team or bereavement program exists. Currently, we are a team of four music therapists working at the hospital, providing our service at the Adult, Paediatric and Neonatal ICU and at the maternity ward. In the NICU, music therapy is offered from Monday to Friday, with a regular caseload of 8-14 patients per day. At the CPO, music therapy was formalized as part of the hospital's strategy of 'humanization of care' after a successful research project about family-centred music therapy in the NICU (Ettenberger et al., 2014; Ettenberger et al., 2016). End-of-life care (in the NICU and elsewhere) is an integral part of our clinical work, although it is not yet a formalized procedure, and we are just beginning to systematize our efforts in this area. This means that we currently have no guidelines that regulate the referral of dying patients to music therapy, which in the case of the NICU, depends very much on the attending neonatologist. However, it is common practice that once a patient receives a music therapy referral, we continue music therapy services; this includes also cases in which a baby might transition to end-of-life care during hospitalization. The mortality rate in the NICU of the CPO is about $3 \%$ or about 25 to 30 babies per year. Considering that about $80 \%$ of these babies die within their first week of life and are not referred to music therapy, end-of-life care is still a relatively rare event for us, with approximately 4 to 6 cases per year.

\section{Case vignettes}

During the next section, three clinical case vignettes are presented and discussed in relation to rituality, culture, memory making, and family-centred care. Additionally, some of the specific challenges when accompanying parents and babies during end-oflife care in the NICU are outlined.

How music therapy is performed will naturally depend on each music therapist's background, training, and on the organizational and structural realities described above. But also the culture of each country and region - and finally of each NICU needs to be considered. Family structures, social relationships, and of course the music and 'musicking' are all socially and culturally shaped aspects that influence how music therapy in the NICU will be provided. In relation to end-of-life care, also the families' experiences, expectations and preferences regarding mourning and bereavement are important to bear in mind. Since the following case vignettes are derived from our early clinical practice, it is not intended to provide a comprehensive method- 
ological framework for providing music therapy during end-of-life care in the NICU. Rather it is hoped that the cases stimulate further discussion among music therapists and health care professionals concerned with this topic. Therefore, while some of the case vignettes may resonate with the experiences of music therapists in other countries and settings, some might not; thus it is important to recognize and honour the diversity of approaches in the field.

\section{Case vignette 1: Family-centred care and memory making ${ }^{2}$}

Sara was born at 30 weeks with an extremely low birth weight and with a fulminant necrotizing enterocolitis ${ }^{3}$. During her first days of life she required mechanical ventilation and a series of surgeries. Due to the complexity of her case, she was referred to our NICU from another hospital when she was 1 month old. At admission, she had only 10\% of her small intestine left, which meant that her prognosis for survival was poor. The medical staff referred her to music therapy for complementary pain management and end-of-life care. Her mother Jenny struggled very much with the fact that her baby might not survive. She had lost another baby during pregnancy about one year and a half ago.

During the initial sessions I worked with Sara alone, trying to calm her pain and hunger (she could not eat) by vocally reflecting her jerky movements and facial expressions, which I cautiously tried to modulate to a more stable and structured melody towards the end of the session. Soon, one of my music therapy colleagues also started to work with her and sometimes we would work simultaneously with Sara.

During the first sessions with her mother Jenny, we offered receptive experiences, trying to create a holding musical environment that allowed her to be with Sara in a somehow different atmosphere. Slowly Jenny was able to talk with us about how she felt, about her thoughts and difficulties and what kind of music she wished for her baby. She started to propose traditional children's songs or lullabies, which we sang together with her for Sara. In a few occasions we had also the chance to accompany Sara's older sister, who was allowed to visit her a couple of times, and her father. Since the prognosis of Sara was poor, but did not pose an immediate threat to her life, together with Jenny we had the time to compose a variety of songs for her: welcome songs, songs for sleeping, songs for feeding, etc. As the weeks passed by, Sara grew older, but still had difficulties in finding relaxation and calmness due to her constant pain and hunger. We often accompanied her during nursery procedures such as bathing and changing clothes for example but also during invasive procedures such as medical canalizations, taking blood samples, and putting catheters. During these sessions we worked in close collaboration with the staff, who seemed to appreciate music therapy very much and who also developed a strong and loving relationship with Sara and Jenny. One of the head nurses even wrote a song for Sara, which my music therapy colleague recorded with her. On several occasions we suggested to Jenny that we take some pictures and videos during the sessions with the aim to create memories that would hopefully last a lifetime.

Sara died after 6 months in the NICU. Following Jenny's wish, one of the music therapists accompanied the family during the funeral, singing the most important songs of the sessions including the composition made by head nurse. We also collected all the photos and videos and the staff added their own pictures they took with Sara and Jenny. About 2 months after Sara's death, I called Jenny and asked her if she felt ready to be handed over the photos and videos. She agreed and we made an appointment at the hospital. The re-encounter with Jenny was very moving; she was missing Sara a lot and cried frequently. Most of the staff came to see her and together with Jenny we watched the photos and videos of Sara and we exchanged anecdotes and stories about her and remembered her smiles and her big eyes. Jenny was grateful about the photos and videos, and watching them together provided an opportunity for all of us to honour Sara's life together.

For the whole team, the case of Sara and Jenny was very moving and due to her long hospitalization we all formed strong bonds with both of them. Family-centred care approaches can provide a useful framework when considering the integration of caregivers to the music therapy process in hospital settings (e.g. Ettenberger, 2017; Haslbeck, 2016; Shoemark \& Dearn, 2008; Shoemark, 2004) and are particularly im- 
portant during end-of-life care (Kenner, Press, \& Ryan, 2015). Empowerment of parents, shared decision-making, respecting, and honouring the families' traditions and beliefs and considering the family as an essential part in the wellbeing of the babies are all principles that are mentioned equally in recommendations regarding familycentred care and palliative care in the NICU (Gooding et al., 2011; Kenner, Press, \& Ryan, 2015; Kuo et al., 2012; ).

Considering the case of Sara, especially the aspect of shared decision-making was crucial for the development of the music therapy process. The emotional states of family members can fluctuate frequently during end-of-life care and denial, frustration, hope, anger, or resignation, are all common experiences for parents in this situation. These emotional states can influence the level of involvement during the music therapy sessions, because they might shape the family's disposition or willingness for engagement. For us, this meant that the participation of Sara's family was renegotiated constantly and ranged from the active partaking of the parents during songwriting and singing to receptive sessions. At times, Jenny also opted to not participate in music therapy but clearly expressed her wish for us to continue to work with Sara alone. Being able to opt out from music therapy is an offer that we express when initiating the therapy process and is an important anchor for parents when the experiences are too overwhelming; being given the choice whether to participate in music therapy or not can help parents to regain an at least a minimal sense of control over their and their baby's life.

Memory making is another important aspect during end-of-life care and is a central theme for many parents who lost their baby in the NICU (Cortezzo, Sanders, Brownell, \& Moss, 2015; Shelkowitz et al., 2015). The recordings we made during the sessions with Sara - which included audio recordings, video recordings, and taking pictures were essential in trying to create memories that would hopefully last a lifetime. While the use of recordings and memory making are fundamental aspects in many end-of-life care programs ${ }^{4}$, the specific cultural setting and the families' individual belief systems certainly influence if, how, at what moments, and under which circumstances recordings are used. Shortly after Sara's death for example, Jenny let us know that she was not yet ready to receive the pictures and videos, but this changed over time. Based on a personal initiative, I invited Jenny to the hospital 2 months later and after watching together and handing her over the material, she was grateful for the pictures and videos and said: "With these pictures I will never forget her."

In the case of Sara, also the involvement of the staff was highly valuable. In Colombia, people easily build up close social relationships and most Colombians are used to constantly sharing physical as well as personal and intimate spaces. Thus, when a baby dies after long weeks and months of struggles, the staff can also become emotionally affected. Accompanying nursing care tasks such as bathing or changing diapers with music provided a space for interaction and bonding not only for Sara's family but also for the professional team. The lyrics that one of the head nurses wrote for Sara were transformed into a song that became an important element both during our sessions and after Sara's death at the funeral. These interventions were highly appreciated by the NICU staff but are clearly shaped by the social and cultural expectations of interpersonal relationships, the relativity of rules and boundaries and the strong sense of community in Colombian society.

\section{Case vignette 2: Facing the uncertainty of death}

Juan was born at 26 weeks, a birth weight of 675 grams and with multiple difficulties related to his early start in life. Immediately after birth he needed to be ventilated and remained in a critical condition over the next couple of weeks. He was diagnosed with Bronchopulmonary Dysplasia $(B P D)^{5}$ and a damaged bowel. When Juan was 35 days old, he was medically stable and the attending neonatologist referred him to music therapy for supporting his breathing and for fostering bonding with his parents. 
During the first session, both Juan's mother Andrea and his father Felipe were present. Andrea was crying while looking at Juan through the glass of the incubator; Felipe was holding a bible and prayed. During our initial conversation, the parents told me that they belonged to a Christian church and that they had regularly sung for Juan during pregnancy both at their church and at home. These songs - mostly spiritual songs, Christian pop songs, and sung prayers - soon became the central musical themes during our sessions. In occasions that Andrea was able to hold Juan in kangaroo care, we sang some of her favourite Christian pop songs, which we adapted by integrating Juan's name to the lyrics. When Juan needed to stay in the incubator, we created short welcome songs or improvised songs about Andrea's feelings for him. In the meanwhile, Juan's health fluctuated a lot. He got an infection, was intubated, recovered, was taken off mechanical ventilation but then got a further infection and needed to be ventilated once more. These changes in Juan's health were very difficult for Andrea. When he was getting better, she cautiously expressed her hopes and illusions about a life after hospitalization. When his health declined, these hopes were broken. While in many sessions the music was aimed at helping Juan with his breathing and relaxation, picking up and integrating Andrea's current emotional states were equally important. Songwriting, song improvisation, and singing significant pieces of music were all interventions that allowed Andrea to express also some of her more ambiguous feelings and thoughts. In some occasions, she also opted to listen to improvised music, which we played on a nylon-string guitar. In these sessions, musical elements such as harmonic tension-release or modulating between major and minor modalities provided an additional opportunity for musically matching her feelings. Besides the sessions with his parents, we also had the chance to provide procedural support for Juan, for example during one of the extubations or during the use of nebulizers to treat his BPD. With the consent of Andrea and the medical staff, my colleague and I accompanied him even during two long surgeries in which parts of his damaged intestine were removed. This close accompaniment of Juan was highly appreciated by the parents and the professional staff. After 4 months of fighting for his life, Juan was finally getting better; he was growing and even started to bottle-feed. Also the medical team was more optimistic. However, he remained prone to infections and suddenly needed to be ventilated once more. This time, his health declined rapidly and Juan died a couple of days later. On the last day of his life, my music therapy colleague was able to accompany Andrea and Juan for the last time, singing her favourite song "Flying with angels".

The repetitive fluctuations and the uncertainty regarding Juan's health were very challenging for both his parents and the staff. Predicting death in the ICU is very complex and especially with non-cancer diseases it can be problematic for physicians to recognize accurately a dying patient (Meadow et al., 2011; Papadimos et al., 2011). Due to the constant changes of Juan's condition, it was essential for us to maintain excellent communication with the medical staff and to be well informed about his current health status. This helped us to regularly re-evaluate and adapt our clinical goals according to his present state and to sensitively address Andrea or Felipe when offering music therapy or when discussing Juan's development or challenges.

Picking up and integrating the emotional states of his parents to the music therapy sessions was equally significant. Making progress and then suffering a setback can be very frustrating for parents in the NICU and can increase feelings of uncertainty (Bolívar Montes \& Montalvo Prieto, 2016). As music therapists, we tried to counterbalance the sense of helplessness and despair that often accompanied Juan's parents through our constant presence and support, even during surgeries. When Juan was too unstable and we had to suspend music therapy for a while, we consciously tried to engage Andrea and Felipe in short conversations inside and outside the NICU in order to keep track of them and to maintain a close relationship. This was vital for being able to more easily resume music therapy when he was sufficiently stable again. The ups and downs that Juan's parents went through during his hospitalization required also being sensitive to more ambiguous feelings they might have experienced. Song improvisation, songwriting, and trying to musically match their current moods, were some of the interventions used with the aim to acknowledge both 'positively' and 'negatively' associated feelings as normal experiences in such a situation. Being aware of over-iden- 
tification and the dynamics of transference and countertransference were essential in this sense and are important elements in medical music psychotherapy (Mondanaro, 2016).

As stated in the introductory section, about $80 \%$ of the deaths in our NICU occur within the first week of life and these babies are not referred to music therapy. But also a relatively stable baby might get worse or even die in a very short time. At least three or four times, Juan was very close to death during his hospitalization and had to be resuscitated twice. However, at the end he was doing well and we all thought that the worst was over. Then he suddenly deteriorated and died within just a couple of days. This short time period between life and death can be an additional challenge, because parents have not much time to prepare and anticipate their loss. Although unanticipated loss is more frequently mentioned in the literature for perinatal loss including miscarriages or stillbirths (Kenner, Press, \& Ryan, 2015; Kerstin \& Wagner, 2012; Romesberg, 2004), it might well play an important role when also older babies suddenly die in the NICU. Knowing about the families' social support system is paramount in this sense, since support from families and friends, but also an active participation in religious communities for example, can ameliorate complicated grief (CG) reactions after perinatal loss (Kerstin \& Wagner, 2012). Support groups or bereavement services after a baby dies can additionally help parents to cope with their loss (Capitulo, 2005; Harvey, Snowdon, \& Elbourne, 2008), but this is still not common practice in Colombia.

A couple of weeks after Juan's death I tried to contact Andrea by phone. Her mother picked up and told me that Andrea and Felipe were holding up fine and that they were spending most of their time with the church community. Andrea was not answering phone calls at the moment, but her mother told me that she had regularly spoken of music therapy and how important it had been for her and her husband during Juan's hospitalization.

\section{Case vignette 3: Rituality}

Baby Martin was born full-term, but due to a severe perinatal asphyxia and a subsequently developed Hypoxic Ischemic Encephalopathy (HIE), he had to be mechanically ventilated immediately after birth and was treated with moderate hypothermia ${ }^{6}$. After about two weeks, it became clear that Martin had minimal chances of survival. The medical staff informed me of his condition and asked me to work with him and his family. This was shortly after having implemented music therapy in the NICU and Martin was my first patient who was referred to me for end-of-life care.

The same morning, while I was working with another baby, I saw Martin's family arriving at the NICU. The parents entered first but then also Martin's sister. This was unusual, since siblings are normally not allowed to visit the babies and spoke to his critical condition. A couple of minutes later a priest came in and held a short service for Martin and his family. In the meanwhile I finished my session and glimpsed at them from time to time. After the sermon, the priest went out and Martin's family stood around his crib, seemingly helpless and unsure what to do next. I walked towards them and presented myself and asked them if they would like to listen to some music while they were with Martin. Martin's parents agreed immediately - and I felt - somehow relieved. I played a simple harmonic progression with a steady and slow beat and accompanied the guitar playing with an improvised hummed melody, trying to create a calm and holding atmosphere. Soon, Martin's sister started to cry and I remember feeling initially unsure about whether to continue or not. As I kept playing, also the parents started to cry silently and gently touched their baby's hand, feet, and face. After about 15 to 20 minutes, I let the music slowly fade out and stood with them in silence. Then, the parents covered Martin with a blanket they brought with them and a couple of minutes later I saw them leaving the NICU. Martin died the same night.

While this case description is less detailed compared to the other vignettes, it exemplifies a particular part of our clinical practice, in which we see a dying baby and its family for a single session. This can be challenging, because there is neither a previously established therapeutic relationship nor are there any shared musical experiences 
with the families that can help us music therapists to navigate in such a delicate situation. But it can also be an opportunity to create a (musical) atmosphere that hopefully supports parents in connecting with their baby, in honouring its life and to say goodbye.

Music, together with other art forms, has been an integral part of rituals and ceremonies for thousands of years (Dissanayke, 2009). A ritual is a set of coordinated and synchronized behaviours and actions that gain relevance by the way they are performed: "A ritual as ritual has no purpose outside itself: the purpose of the ritual is that it be performed, and performed correctly" (Merker, 2009, p. 46). At its essence, a ritual marks an important event or moment in our lives, whether the goal may be to worship, honour, thank, celebrate, welcome, or farewell. This means that rituals "provide meaning and order to transitions, and symbolically connect people and events" (Kobler, Limbo, \& Kavanaugh, 2007, p. 299). While our daily life is full of rituals, in the NICU, however, this is different. Parents normally do not celebrate their baby's birth, as they would probably do it at home. Often, they cannot share their joy with their families and friends and even the mere thought of celebration can be difficult to bear considering the amount of stress, worries, and anxiety that many parents face in the NICU. The same is true when a baby dies. Saying good-bye is often a silent and solitary act in the NICU, without music, poetry, or the presence of friends or even important family members. Rituals can thus be crucial elements during end-of-life care in the NICU and can help families to find support, meaning and to initiate their grief process in the context of a structured and shared experience (Capitulo, 2005; Kobler, Limbo, \& Kavanaugh, 2007).

While the (exact) reproduction of learned actions and behaviours is certainly an important aspect in defining rituals (Merker, 2009), at least in the context of our clinical practice, these actions and behaviours are however often improvised. Since in our hospital no palliative care team or bereavement program exists, there is no established way of working with parents and babies in such a situation; there is no formalized 'ritual' that might guide and support parents in expressing their grief, no ceremonial acts, no rules to follow, no predetermined music dedicated to honour the baby's short but important life. This can be especially challenging considering patients like Martin, since there is no time to discuss or prepare potential rituals that parents might want to be part of. While it remains unclear if Martin's family perceived the music therapy session as a ritual, being accompanied by music in such a situation might have helped to create a context with ritual-like characteristics. Kobler, Limbo, \& Kavanaugh (2007, p. 291) described three dimensions of rituals in perinatal and paediatric death, labelled as meaning-making, intention, and participation.

Meaning-making refers to the inherent goal of any ritual to mark a moment or situation as something significant and meaningful. Symbols such as words, objects, movements, but also music, are used to individually create meaning for the participants and facilitate the grief process. Especially in single sessions like the one with Martin, music therapy is often an unexpected and extraordinary event for families in such circumstances. This can eventually help to adhere a special meaning to the situation, to mark it as something important:

One could say, in fact, that ceremonies composed of music and associated arts are the behavioural or expressive counterpart of religious doctrine and belief, providing something 'special' (shaped, embellished) to do for humans cognisant of and attempting to cope with the problems and uncertainties of mortal existence, whether past, present or future (Dissanayake, 2009, p. 24).

While intention refers to an inner "determination to act in a certain way" (Kobler, Limbo, \& Kavanaugh, 2007, p. 291), participation in rituals for dying babies in the NICU should be voluntary and adjusted to the level of comfort of the parents. In our experience, music is something that parents generally welcome during end-of-life care in the NICU and while levels of participation can vary, music therapy can facilitate the synchronization of the families' behaviours and actions during a session and thus to 
"coordinate temporally structured events through interaction" (Clayton, Sager, \& Will, 2004, p. 3). Entrainment - the conscious synchronization and modification of musical elements in relation to physiological, emotional or behavioural states of the families and babies - can be especially useful in this sense; both entrainment and synchronization are core aspects in many rituals (Clayton, Sager, \& Will, 2004; Merker, 2009).

Whether prepared and anticipated or improvised in the moment, music can play a crucial role in establishing a ritual for families during end-of-life care in the NICU. And although there are no testimonies from Martin's family about their perception of music therapy, I had the strong impression that the music helped them to instinctively do what they needed to do - to say goodbye.

\section{Conclusions}

In the three case illustrations, I provided some early clinical insights into music therapy during end-of-life care in the NICU. While it was aimed at highlighting particular issues in each vignette, family-centred care, rituality or memory making are certainly transversal themes during end-of-life care in general and not limited to single experiences. Similarly, context and culture are underlying forces that influenced all the cases and are particularly important to bear in mind when working with families whose background differ from those of the music therapist (Forrest, 2011, 2014; Mondanaro, 2016). In addition to cultural differences, mothers and fathers can experience distinct challenges when having a baby in the NICU (Mondanaro, Ettenberger, \& Park, 2016), which may result in different ways of coping when facing the death of their baby (Capitulo, 2005; Kersting \& Wagner, 2012). Thus, developing a selective understanding of grief and loss in parents is crucial for end-of-life care in the NICU but also vital with respect to other causes than death (Dyer, 2005a, b). Family-centred care principles, adapting a sensitive language in communicating with families and individualizing the therapy offer according to the patients and families needs and expectations are certainly paramount aspects in this sense (Kenner, Press, \& Ryan, 2015; Mullen, Reynolds, \& Larson, 2015).

Considering that our clinical work takes place in Colombia, it is also important to recognize the subtle differences in general end-of-life care approaches between Latin America and North America or Europe. A study carried out in California for instance (California Health Care Foundation, 2006), found significant differences among ethnic groups regarding life-sustaining treatments with $44 \%$ of Latino and African-American participants believing that "everything possible should be done in all circumstances to save a life" in contrast to $14 \%$ of non-Hispanic white participants. Fajardo et al. (2012) analysed the end-of-life decisions in NICUs of five Latin American countries and concluded that withholding or withdrawing of life support is very rare in Latin America and that much more deaths are accompanied by full life support and Cardiopulmonary Resuscitation (CPR) compared to the United States, Canada, or European countries. Also, there seem to be less family involvement when it comes to decisions regarding withholding life-sustaining treatments. Such distinctions are important to acknowledge, since they reflect also wider cultural, social, and religious values (Luce, 2010).

Being able to accompany and support babies and their families during their last stages of life is a huge honour and can be very rewarding. However, it is also challenging and at times frustrating and the need for staff support and to recognize staff suffering is frequently mentioned in guidelines regarding end-of-life care (Cook \& Rocker, 2014; Kenner, Press, \& Ryan, 2015; Papadimos et al., 2011). Adequate training, close supervision and cultivating personal wellbeing can help music therapists to cope when working with dying babies and their families in the NICU. In our own case, weekly team meetings provide an additional opportunity to informally discuss some of the difficulties and challenges we experience when working with such patients.

As stated in the introduction of this section, it is important to point out that end-oflife care in our hospital is not yet a formalized process and no palliative care team or bereavement program exists. Thus, music therapy is still provided sporadically in this 
field and we are just beginning to systematize our work in this area. Any conclusions for clinical practices outside our own specific setting need therefore to be drawn with great care. However, we are keen to learn from other music therapists working in this field and hope that this article stimulates the exchange of experiences regarding music therapy during end-of-life care in the NICU.

\section{Acknowledgements}

I want to thank Dr. Helen Shoemark for her comments and suggestions in writing this article.

\section{Notes}

1. All the names in the case vignettes were changed and consent for presenting the cases was obtained.

2. This case vignette was originally published in Spanish in Ettenberger (2017b). Consent for publishing it in this article was obtained.

3. Necrotizing enterocolitis is a complex bowel condition of very preterm babies, in which parts of the bowel undergo tissue death.

4. See for example the wonderful work of music therapist Brian Schreck at Cincinnati Children's Hospital, who records heartbeats of palliative care patients and their families and uses these recordings for songwriting (http://blog.cincinnatichildrens.org/patient-family-experience/and-the-beat-goes-on).

5. Bronchopulmonary Dysplasia (BPD) is a chronic lung disease common in extremely preterm infants and includes abnormal findings on chest x-ray and the need for supplemental oxygen at $\geq 28$ days of life (Sahni \& Phelps, 2011).

6. Hypoxic Ischemic Encephalopathy (HIE) is a type of brain damage that occurs when the brain does not receive enough oxygen and blood. Moderate hypothermia consists of selectively cooling the head or whole body of the baby as a treatment of the condition (Allen, 2014).

\section{References}

AAP American Academy of Pediatrics. (2013). Pediatric palliative care and hospice care commitments, guidelines, and recommendations. Pediatrics, 132(5), 966-972.

http://dx.doi.org/10.1542/peds.2013-2731

AAP American Academy of Pediatrics. (2000). Palliative care for children. Pediatrics, 106(2), 351-357.

Allen, K. A. (2014). Moderate hypothermia: Is selective head cooling or whole body cooling better? Advances in Neonatal Care, 14(2), 113-118, http://dx.doi.org/10.1097/ ANC.0000000000000059.

Archie, P., Bruera, E., \& Cohen, L. (2013). Music-based interventions in palliative cancer care: A review of quantitative studies and neurobiological literature. Support Care Cancer, 21, 2609-2624, http://dx.doi.org/10.1007/s00520-013-1841-4.

Bieleninik, L., Ghetti, C., \& Gold, C. (2016). Music therapy for preterm infants and their parents: A meta-analysis. Pediatrics, 138(3), http://dx.doi.org/10.1542/peds.2016-0971.

Bolívar Montes, L. A., \& Montalvo, A. (2016). Uncertainty associated to parents of preterm infants hospitalized in neonatal intensive care units. Investigación y Educación en Enfermería, 34(2), 360-367, http://dx.doi.org/10.17533/udea.iee.v34n2a16.

Bradt, J., \& Dileo, C. (2010). Music therapy for end-of-life care. Cochrane Database of Systematic Reviews, 1, CD007169, http://dx.doi.org/10.1002/14651858.CD007169.pub2.

California Health Care Foundation. (2006). Attitudes towards end-of-life care in California. Retrieved from http://www.chcf.org/publications/2006/11/attitudes-toward-endoflifecare-in-california. 
Capitulo, K. L. (2005). Evidence for healing interventions with perinatal bereavement. The American Journal of Maternal/Child Nursing, 30(6), 389-396, https://doi.org/10.1097/ 00005721-200511000-00007.

Cook, D., \& Rocker, G. (2014). Dying with dignity in the intensive care unit. New England Journal of Medicine, 370, 2506-2514, http://dx.doi.org/10.1056/NEJMra1208795.

Cortezzo, D. E., Sanders, M. R., Brownell, E. A., \& Moss, K. (2015). End-of-life care in the neonatal intensive care unit: Experiences of staff and parents. American Journal of Perinatology, 32(8), 713-724, http://dx.doi.org/10.1055/s-0034-1395475.

Clayton, M., Sager, R., \& Will, U. (2004). In time with the music: The concept of entrainment andits significance for ethnomusicology. ESEM CounterPoint, 1, 1-45.

Dissanayake, E. (2009). Root, leaf, blossom, or bole: Concerning the origin and adaptive function of music. In S. Malloch \& C. Trevarthen (Eds.), Communicative musicality. Exploring the basis of human companionship (pp. 17-30). Oxford, England: Oxford University Press.

Dyer, K. A. (2005a). Identifying, understanding, and working with grieving parents in the NICU, part I: Identifying and understanding loss and the grief response. Neonatal Network, 24(3), 35-46.

Dyer, K. A. (2005b). Identifying, understanding, and working with grieving parents in the NICU, part II: Strategies. Neonatal Network, 24(4), 27-40.

Ettenberger, M. (2017a). Music therapy in the neonatal intensive care unit (NICU): Putting the families at the centre of care. British Journal of Music Therapy, 1-6, http://dx.doi.org/ $10.1177 / 139457516685881$.

Ettenberger, M.. (2017b). Musicoterapia en la Unidad de Cuidados Intensivos Neonatal - Un enfoque centrado en la familia. [Music therapy in the neonatal intensive care unit - A family-centred approach]. In G. Federico \& V. Tosto G (Eds.), Lo que suena en las sesiones. Musicoterapia, casos clínicos. Buenos Aires, Argentina: Editorial Kier. In press.

Ettenberger, M., Rojas, C., Odell-Miller, H., \& Parker, M. (2016). Family-centred Music Therapy with preterm infants and their parents in the neonatal intensive care unit (NICU) in Colombia - A mixed-methods study. Nordic Journal of Music Therapy, http://dx.doi.org/ 10.1080/08098131.2016.

Ettenberger, M., Odell-Miller, H., Rojas, C., Torres, S., Parker, M., \& Camargo, S. M. (2014). Music therapy with premature infants and their caregivers in Colombia - A mixed methods pilot study including a randomized trial. Voices: A World Forum for Music Therapy, 14(2), http://dx.doi.org/10.15845/voices.v14i2.756.

Fajardo, C. A., González, S., Zambosco, G., Cancela, M. J., Forero, L. V., Venegas, M., et al., \& Lantos, J. (2012). End of life, death and dying in neonatal intensive care units in Latin America. Foundation Acta Padiatrica, 101, 609-613, http://dx.doi.org/10.1111/ j.1651-2227.2012.02596.x.

Flyvbjerg, B. (2006). Five misunderstandings about case-study research. Qualitative Inquiry, 12(2), 219-245.

Forrest, L. (2014). Your song, my song, our song: Developing music therapy programs for a culturally diverse community in home-based paediatric palliative care. Australian Journal of Music Therapy, 25, 15-27.

Forrest, L. (2011). Supportive cancer care at the end of life: Mapping the cultural landscape in palliative care and music therapy. Music and Medicine, 3(1), 9-14.

Gooding, J. S., Cooper, L. G., Blaine, A. I., Franck, L. S., Howse, J. L., \& Berns, S. D. (2011). Family support and family-centered care in the neonatal intensive care unit: Origins, advances, impact. Seminars in Perinatology, 35(1), 20-28, http://dx.doi.org/10.1053/ j.semperi.2010.10.004.

Gutgsell, K. J., Schluchter, M., Margevicius, S., DeGolia, P. A., McLaughlin, B., Harris, M., et al., \& Wiencek, C. (2013). Music therapy reduces pain in palliative care patients: A randomized controlled trial. Journal of Pain and Symptom Management, 45(5), 822-831, http://dx.doi.org/10.1016/j.jpainsymman.2012.05.008. 
Hartling, L., Shaik, M. S., Tjosvold, L., Leicht, R., Liang, Y., \& Kumar, M. (2009). Music for medical indications in the neonatal period: A systematic review of randomised controlled trials. Archives of Diseases in Childhood. Fetal and Neonatal Edition, 94, 349-354, http://dx.doi.org/10.1136/adc.2008.148411.

Harvey, S., Snowdon, C., \& Elbourne, D. (2008). Effectiveness of bereavement interventions in neonatal intensive care: A review of the evidence. Seminars in Fetal \& Neonatal Medicine, 13, 341-356, http://dx.doi.org/10.1016/j.siny.2008.03.011.

Haslbeck, F. (2016). Three little wonders. Music therapy with families in neonatal care. In S. Lindhal (Ed.), Music Therapy with Families (pp. 19-44). London, England: Jessica Kingsley Publishers.

Haslbeck, F. (2013). The interactive potential of creative music therapy with premature infants and their parents: A qualitative analysis. Nordic Journal of Music Therapy, 23(1), 36-70, http://dx.doi.org/10.1080/08098131.2013.790918.

Haslbeck, F. (2012). Music therapy for premature infants and their parents: an integrative review. Nordic Journal of Music Therapy, 21(3), 1-38, http://dx.doi.org/10.1080/ 08098131.2011.648653.

Hilliard, R. E. (2005). Music therapy in hospice and palliative care: A review of the empirical data. eCAM, 2(2), 173-178, http://dx.doi.org/10.1093/ecam/neh076.

Hodges, A. L., \& Wilson, L. L. (2010). Preterm infants' responses to music: An integrative literature review. Southern Online Journal of Nursing Research, 10(3), http://www.snrs.org.

Horne-Thompson, A., \& Grocke, D. (2008). The effect of music therapy on anxiety in patients who are terminally ill. Journal of Palliative Medicine, 11, 582-590.

Kenner, C., Press, J., \& Ryan, D. (2015). Recommendations for palliative and bereavement care in the NICU: A family-centered integrative approach. Journal of Perinatology, 35, 19-23, http://dx.doi.org/10.1038/jp.2015.145.

Kersting, A., \& Wagner, B. (2012). Complicated grief after perinatal loss. Dialogues in Clinical Neuroscience, 14(2), 187-194.

Kobler, K., Limbo, R., \& Kavanaugh, K. (2007). Meaningful moments. The use of ritual in perinatal and pediatric death. The American Journal of Maternal/Child Nursing, 32(5), 288-295.

Kordovan, S., Preissler, P., Kamphausen, A., Bokemeyer, C., \& Oechsle, K. (2016). Prospective study on music therapy in terminally ill cancer patients during specialized inpatient palliative care. Journal of Palliative Medicine, 19(4), 394-399, https://doi.org/10.1089/ jpm.2015.0384.

Krueger, C. (2010). Exposure to maternal voice in preterm infants: A review. Advances in Neonatal Care, 10(1), 13-20, http://dx.doi.org/10.1097/ANC.0b013e3181cc3c69.

Kuo, D. Z., Houtrow, A. J., Arango, P., Kuhlthau, K. A., Simmons, J. M., \& Neff, J. M. (2012). Family-centered care: Current applications and future directions in pediatric health care. Maternal \& Child Health Journal, 16, 297-305, http://dx.doi.org/10.1007/ s10995-011-0751-7.

Luce, J. M. (2010). End-of-life decision making in the intensive care unit. American Journal of Respiratory Critical Care Medicine, 182, 6-11, http://dx.doi.org/10.1164/ rccm.201001-0071CI.

Mancini, A., Uthaya, S., Beardsley, C., Wood, D., \& Modi, N. (2014). Practical guidance for the management of palliative care on neonatal units. $R C P C H$, Retrieved from, http://www.rcpch.ac.uk/news/palliative-care-neonatal-units-first-guidance-published-uk.

McConnell, T., Scott, D., \& Porter, S. (2016). Music therapy for end-of-life care: An updated systematic review. Palliative Medicine, 30(9), 877-883, http://dx.doi.org/10.1177/ 0269216316635387.

Meadow, W., Pohlman, A., Frain, L., Ren, Y., Kress, J. P., Teuteberg, W., \& Hall, J. (2011). Power and limitations of daily prognostications of death in the medical intensive care unit. Critical Care Medicine, 39(3), 474-479, http://dx.doi.org/10.1097/CCM.0b013e318205df9b. 
Merker, B. (2009). Ritual foundations of human umiqueness. In S. Malloch \& C. Trevarthen (Eds.), Communicative musicality. Exploring the basis of human companionship (pp. 45-60). Oxford, England: Oxford University Press.

Mondanaro, J. (2016). Multiculturally focused medical music psychotherapy in affirming identity to facilitate optimal coping during hospitalization. Music Therapy Perspectives, 34, 154-160, https://doi.org/10.1093/mtp/miw019.

Mondanaro, J., Ettenberger, M., \& Park, L. (2016). Mars rising: Music therapy and the increasing presence of fathers in the NICU. Music \& Medicine, 8(3), 96-107.

Mullen, J. E., Reynolds, M. R., \& Larson, J. S. (2015). Caring for pediatric patients' families at the child's end of life. Critical Care Nurse, 35(6), 46-55, http://dx.doi.org/http://dx.doi.org/ $10.4037 / \operatorname{ccn} 2015614$.

National Consensus Project for Quality Palliative Care. (2013). Palliative care clinical practice guidelines for quality palliative care (3rd ed.). Retrieved from http://www.nationalconsensusproject.org/NCP_Clinical_Practice_Guidelines_3rd_Edition.pdf

NHS. (2016). End of life care. Retrieved from http://www.nhs.uk/Planners/end-of-life-care/ Pages/what-it-involves-and-when-it-starts.aspx

OKelly, J. (2002). Music therapy in palliative care. International Journal of Palliative Nursing, $8(3), 130-136$

Papadimos, T. J., Maldonado, Y., Tripathi, R. S., Kothari, D. S., \& Rosenberg, A. L. (2011). An overview of end-of-life issues in the intensive care unit. International Journal of Critical Illness and Injury Science, 1(2), 138-146, http://dx.doi.org/10.4103/2229-5151.84801.

Romesberg, T. L. (2004). Understanding grief: A component of neonatal palliative care. Journal of Hospice and Palliative Nursing, 6(3), 161-170.

Sahni, J., \& Phelps, S. J. (2011). Nebulized furosemide in the treatment of bronchopulmonary dysplasia in preterm infants. Journal of Pediatric Pharmacology and Therapeutics, 16(1), 14-22.

Shelkowitz, E., Vessella, S. L., O’Reilly, P., Tucker, R., \& Lechner, B. E. (2015). Counseling for personal care options at neonatal end of life: A quantitative and qualitative parent survey. BMC Palliative Care, 14, 1-11, http://dx.doi.org/10.1186/s12904-015-0063-6.

Shoemark, H., \& WCMT 2014 NICU Music Therapy Roundtable Group. (2015). culturally transformed music therapy in the perinatal and paediatric neonatal intensive care unit: An international report. Music \& Medicine, 7(2), 35-36.

Shoemark, H., \& Daern, T. (2008). Keeping parents at the centre of family centred music therapy with hospitalized infants. Australian Journal of Music Therapy, 19, 3-24.

Shoemark, H. (2004). Family-centered music therapy for infants with complex medical and surgical needs. In M. Nöcker-Ribaupierre (Ed.), Music therapy for premature and newborn infants (pp. 141-158). Gilsum, NH: Barcelona Publishers.

Standley, J. M. (2012). Music therapy research in the NICU: An updated meta-analysis. Neonatal Network, 31(5), 311-316, http://dx.doi.org/10.1891/0730-0832.31.5.311.

Truog, R. D., Campbell, M. L., Curtis, J. R., Haas, C. E., Luce, J. M., Rubenfeld, G. D., \& Kaufman, D. C. (2008). Recommendations for end-of-life care in the intensive care unit: A consensus statement by the American College of Critical Care Medicine. Critical Care Medicine, 36(3), 953-963, http://dx.doi.org/10.1097/CCM.0B013E3181659096.

Ullsten, A., Eriksson, M., Klässbo, M., \& Volgsten, U. (2017). Live music therapy with lullaby singing as affective support during painful procedures: A case study with microanalysis. Nordic Journal of Music Therapy, 26(2), 142-166, http://dx.doi.org/10.1080/ 08098131.2015.1131187.

van der Heijden, M. J. E., Oliai, S., Jeekel, J., Reiss, I. K. M., Hunink, M. G. M., \& van, M. (2016). Do hospitalized premature infants benefit from music interventions? A systematic review of randomized controlled trials. PLOS ONE, 11(9), e0161848, http://dx.doi.org/ 10.1371/journal.pone.0161848.

WHO. (2016). WHO definition of palliative care. Retrieved from http://www.who.int/cancer/ palliative/definition/en/ 